\title{
Structural Activity of Unsaturated Alcohols and Oxidation Towards Pyrazinium Chlorochromate
}

\author{
K. ANBARASU and K. K. ILAVENIL* \\ Department of Chemistry, Arignar Anna Government Arts College, Affliliated to Bharathidasan University, \\ Trichy, Musiri-621 211, Tamilnadu, India. \\ *Corresponding E-mail: arasu007@gmail.com, illavenil81@gmail.com
}

http://dx.doi.org/10.13005/ojc/350156

(Received: August 16, 2018; Accepted: January 29, 2019)

\begin{abstract}
The kinetics of oxidation of allyl, crotonyl, styryl carbinol by pyrazinium chlorochromate (PzCC) has been investigated in 60\% acetic acid medium. The order was found to be first order with respect to [oxidant], [substrate] and $\left[\mathrm{H}^{+}\right.$.]. The rate of the oxidant decreased with increase in [PzCC] and increased with increase in the percentage of acetic acid. The addition of sodium perchlorate did not show any change in rate constant. Based on the kinetic results, the mechanism between unsaturated alcohol and chromium (VI) oxidant is proposed. The reactivity of unsaturated alcohols was observed to be allyl alcohol< crotonyl alcohol< styryl carbinol.
\end{abstract}

Keywords: Unsaturated alcohols, Oxidation, Kinetics, Sodium perchlorate, Pyrazinium chlorochromate, Mechanism.

\section{INTRODUCTION}

The alcohols on oxidation give acids, aldehydes and ketones based on the nature of the oxidant. They are used largely in perfumes, dye industries, rubber, polymer industry, pharmaceutical industry and agrochemical industry. In synthetic organic chemistry, the oxidation of alcohol to carbonyl group is of greater importance ${ }^{1,2}$. The metal based oxidants like $\mathrm{KMnO}_{4}, \mathrm{Cr}(\mathrm{VI}), \mathrm{RuO}_{4}$, produce large amounts of toxic end-products ${ }^{3,4}$. Chromium (VI) $\left(\mathrm{Cr}^{+}\right)$are powerful oxidizing agent for the oxidation of varieties of organic compounds ${ }^{5}$. Allyl alcohol is used as raw material for the preparation of resins, plasticizers, pharmaceuticals and many organic compounds ${ }^{6}$. Styryl carbinol (Cinnamyl alcohol) is employed as an additive in cosmetic products, soaps, toothpaste, deodorants, as food additives in chewing gum, bakery products, candy and soft drinks.

In order to avoid the versatality of these oxidants, mild $\mathrm{Cr}^{+6}$ derivatives are synthesized from heterocyclic halochromate. Kinetics of oxidation of alcohols using pyridinium chlorochromate ${ }^{7}$, pyridinium fluorochromate $^{8}$, bipyridinium chlorochromate ${ }^{9}$, halosilanes chromium trioxide ${ }^{10}$, quinolinium

This is an Open Access article licensed under a Creative Commons license: Attribution 4.0 International (CC- BY). Published by Oriental Scientific Publishing Company @ 2018 
dichromate ${ }^{11}$, imidazolium dichromate ${ }^{12}$, quinaldinium fluorochromate ${ }^{13}$, quinaldinium dichromate ${ }^{14}$ and quinolinium fluorochromate ${ }^{15}$ and benzyl triethylammonium chlorochromate ${ }^{16}$ has been reported in the literature. The kinetics and oxidation of allyl alcohol, crotonyl alcohol and styryl carbinol using pyrazinium chlorochromate has not yet been investigated. The aim of the work is to study the kinetics of oxidation of unsaturated alcohols by pyrazinium chlorochromate and explain the structural behaviour of these compounds.

\section{MATERIALS AND METHODS}

Allyl alcohol, crotonyl alcohol, styryl carbinol of AR grade were obtained from Sigma Aldrich and used as such. The solvent glacial acetic acid was distilled with chromic oxide and the boiled fraction was collected between $116-118^{\circ} \mathrm{C}$. Doubly distilled water was used throughout the experiment.

\section{Preparation of Pyrazinium chlorochromate (PzCC) $\mathrm{C}_{4} \mathrm{~N}_{2} \mathrm{H}_{5} \mathrm{CrO}_{3} \mathrm{Cl}$}

Pyrazinium chlorochromate (PzCC) was prepared by literature method ${ }^{17}$ as shown below. $0.35 \mathrm{~g}$ of pyrazine in $0.4 \mathrm{ml}$ of $\mathrm{HCl}$ was added along with $8 \mathrm{ml}$ of water. $0.45 \mathrm{~g}$ of $\mathrm{CrO}_{3}$ was dissolved in the mixture of $0.4 \mathrm{ml} \mathrm{HCl}$ and $4 \mathrm{ml}$ of water and added to the above solution and then it was stirred in ice bath for one hour. A bright orange coloured solid was obtained as shown in the reaction (1) below and recrystallised with acidified water.

\section{Kinetic studies}

The unsaturated alcohols were maintained in large excess concentration than the oxidant to attain pseudo-first order conditions. The reactions were carried out at constant temperature using thermostat. The absorbance was recorded using colorimeter at $470 \mathrm{~nm}$, Elico CL-63 Photometer. The pseudo-first order rate constants kobserved were determined up to $75 \%$ completion of the reaction from the linear plots, $r=0.990$ to 0.999 for log [absorbance] versus time.

\section{Correlation analysis}

Linear regression( $r$ ) analysis was carried using computer software, Micro cal origin. The reproducibility of the rate constants was approximately $\pm 2 \%$.

\section{Stoichiometry and Product analysis}

Pyrazinium chlorochromate (PzCC) was added in excess to carry out stoichiometric study. The alcohols and PzCC were mixed in the ratio $1: 2$ and were kept over night at $298 \mathrm{~K}$ in perchloric acid, acetic acid and water mixture. 1 mole of an alcohol consumed 2 moles of PzCC showing 1: 2 stoichiometries. From iodometric methods, the unreacted pyrazinium chlorochromate was determined.The reaction is shown in the equation (2).

Unsaturated alcohol PzCC Aldehyde $\left(\mathrm{R}=-\mathrm{H},-\mathrm{CH}_{3} \&-\mathrm{C}_{6} \mathrm{H}_{5}\right)$

The products acrolein, crotonyl aldehyde and cinnamaldehyde were obtained on oxidation of allyl alcohol, crotonyl alcohol and styryl carbinol as shown in the equation 2. The aldehydes were identified by spot test ${ }^{18}$ as silver mirror test. The aldehyde shown in the above reaction was characterised by IR spectra and Chromatographic method (TLC).

\section{RESULTS AND DISCUSSION}

The kinetics of oxidation of allyl alcohol was studied in $60 \%$ acetic acid and water medium in presence of perchloric acid at constant temperature.

\section{Effect of varying [Alcohol]}

The reaction rate increased linearly with increase in the concentration of alcohols (Table.1). The plot of $\log k_{1}$ versus log [alcohols], (Fig. 1) gave the unit slope $B=0.876$ with linear correlation of $r=0.994$ (allyl alcohol). The experiment was carried for crotonyl alcohol as mentioned above and the unit slope $B=0.882$ with linear correlation of $r=0.998$ was obtained. Styryl carbinol was taken as the substrate and the same procedure was followed as mentioned above. The slope value $B=0.958$ was attained with a linear correlation of $r=0.999$.

\section{Effect of varying [PzCC]}

The concentration of pyrazinium chlorochromate was varied at constant substrate (alcohol) and perchloric concentration. From the plot of log absorbance versus time a linear graph was obtained proving the reaction to be first order with respect to PzCC. The increase in PzCC concentration showed decrease in rate constant value $^{19}$. From the plot of $1 / \mathrm{k}_{1}$ versus $1 /[\mathrm{PzCC}]$ (Fig. 2) a linear graph was obtained since the total $\mathrm{Cr}^{+6}$ was in the form of acid chromate in which is the effective oxidant ${ }^{20}$ and the two nitrogen atom have 
withdrawn the electrons which have resulted in the decrease of rate constant values.
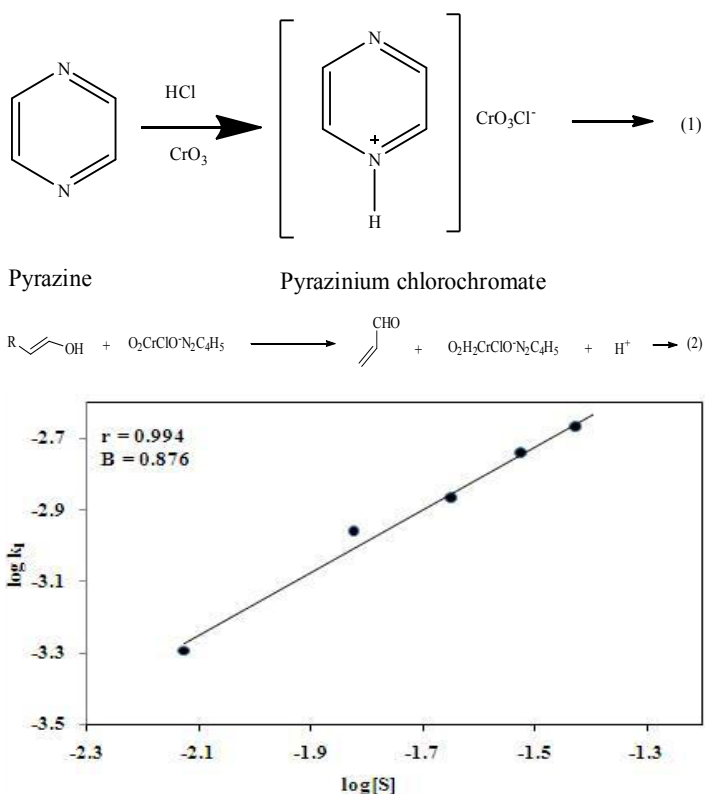

Fig. 1. Plot of $\log k_{1}$ versus $\log$ [alcohols]

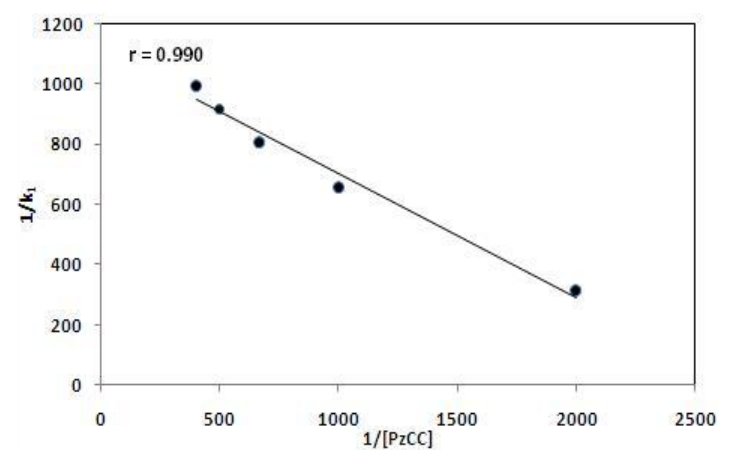

Fig. 2. Plot of $1 / \mathrm{k} 1$ versus $1 /$ [PzCC]

Table.1: Rate data for the oxidation of allyl alcohol by $\mathrm{PzCC}$ at 303k

\begin{tabular}{cccc}
\hline $\begin{array}{c}10^{3}[\mathrm{PzCC}] \\
\left(\mathrm{mol} \mathrm{dm}^{-3}\right)\end{array}$ & $\begin{array}{c}10^{2}[\text { Allyl alcohol }] \\
\left(\mathrm{mol} \mathrm{dm}^{-3}\right)\end{array}$ & $\begin{array}{c}10^{1}\left[\mathrm{H}^{+}\right] \\
\left(\mathrm{mol} \mathrm{dm}^{-3}\right)\end{array}$ & $\begin{array}{c}10^{4} \mathrm{k}_{1} \\
\left(\mathrm{~s}^{-1}\right)\end{array}$ \\
\hline 0.5 & 1.50 & 1.0 & 31.83 \\
1 & 1.50 & 1.0 & 15.23 \\
1.5 & 1.50 & 1.0 & 12.37 \\
2.0 & 1.50 & 1.0 & 10.91 \\
2.5 & 1.50 & 1.0 & 10.04 \\
2.0 & 0.75 & 1.0 & 5.11 \\
2.0 & 2.25 & 1.0 & 13.54 \\
2.0 & 3.00 & 1.0 & 18.17 \\
2.0 & 3.75 & 1.0 & 21.47 \\
2.0 & 1.50 & 0.5 & 6.21 \\
2.0 & 1.50 & 1.5 & 17.29 \\
2.0 & 1.50 & 1.8 & 21.04 \\
2.0 & 1.50 & 1.9 & 22.59 \\
\hline $\mathrm{AcOH}: \mathrm{H}_{2} \mathrm{O}=60: 40(\mathrm{v} / \mathrm{v})$ & Temperature=303K
\end{tabular}

\section{Effect of varying the [Perchloric acid]}

The [perchloric acid] $\mathrm{H}^{+}$was varied in the range of $0.5 \times 10^{-1}$ to $2.5 \times 10^{-1} \mathrm{~mol} \mathrm{dm}^{-3}$ and the rate constants were measured (Table 1). The increase in [perchloric acid] in the oxidation reaction increases the rate and confirms to be first order reaction. From the plot of $\log \mathrm{k}_{1}$ against $\log \left[\mathrm{H}^{+}\right]$a linear graph was obtained with unit slope (Figure 3).

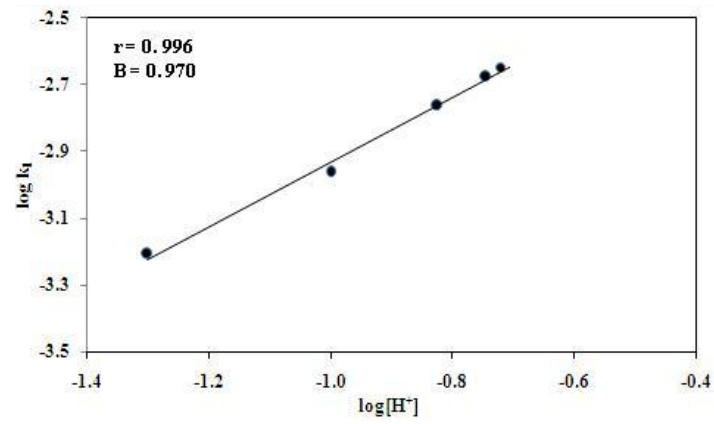

Fig. 3. Plot of logk1 versus $\log \left[\mathrm{H}^{+}\right]$

\section{Effect of varying lonic strength $\left[\mathrm{NaClO}_{4}\right]$}

The effect of sodium perchlorate salt by varying concentration from 0.002 to $0.008 \mathrm{M}$ shown in the Table 2 was studied in Debye-Huckel limit. The rate of the reactions was nearly constant and it implies the ionic interactions in the rate determining step.

\section{Effect of Acetic acid and Water}

The acetic acid composition was varied at six different combinations with water by having the other variables constant. The rate increased with increase in the solvent concentration due to the interaction of ionic species in the slow step, specified in the Table 2. The plot of log $\mathrm{k}_{1}$ versus $1 / \mathrm{D}$ was linear with positive slope ${ }^{21}$ as shown in the Figure 4.

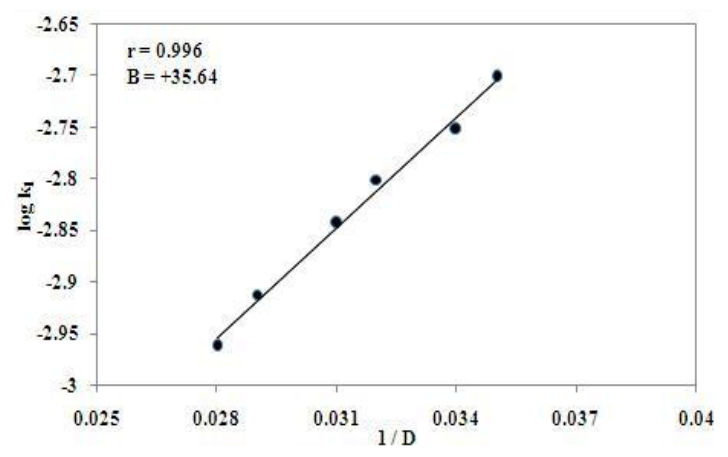

Fig. 4. Plot of log k1 versus 1/D

\section{Free radicals Test}

The addition of acrylonitrile had no effect on the rate of oxidation of allyl alcohol, crotonyl alcohol 
and styryl carbinol. One electron transfer of oxidation is not observed.

\section{Effect of varying $\mathrm{MnSO}_{4}$ concentration}

Addition of $\mathrm{Mn}^{2+}$ in the range of 0.002 to $0.008 \mathrm{M}$ retards the oxidation rate indicating two electron oxidation ${ }^{22}$ given in the Table 2 . This is due to involvement of chromium (IV) intermediate.

\section{Mechanism and Rate law}

From the kinetic investigations, the following mechanism has been proposed and suitable rate law is derived.

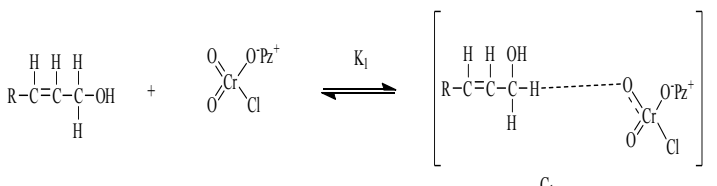

$$
\begin{aligned}
& \mathrm{C}_{1}
\end{aligned}
$$

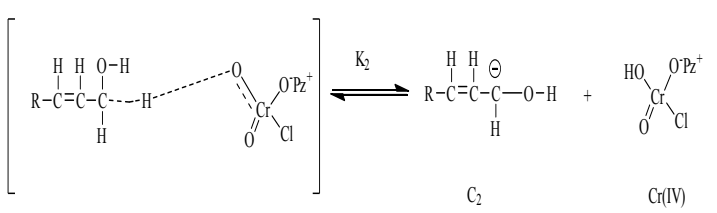

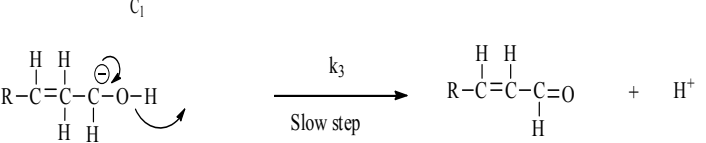

$$
\begin{aligned}
& \mathrm{C}_{2} \quad \text { Aldehyde }
\end{aligned}
$$

$\mathrm{R}=-\mathrm{H}$ (Allyl), $-\mathrm{CH}_{3}$ ( Crotonyl), $-\mathrm{C}_{6} \mathrm{H}_{5}$ ( Styryl)

$$
\mathrm{C}_{1} \text { (Intermediate state) } \quad \mathrm{C}_{2} \text { (Carbanion) }
$$

\section{Rate law}

Rate $=\mathrm{k}_{3}$ [Product $]$

$$
=\mathrm{k}_{3} \mathrm{~K}_{2}[\mathrm{C} 1]
$$

$$
\begin{aligned}
& \quad=\mathrm{k}_{3} \mathrm{~K}_{2} \mathrm{~K}_{1}[\mathrm{~S}][\mathrm{PzCC}]\left[\mathrm{H}^{+}\right] \\
& -\mathrm{d}[\mathrm{PzCC}]=\mathrm{k}_{3} \mathrm{~K}_{2} \mathrm{~K}_{1}[\mathrm{~S}][\mathrm{O}]\left[\mathrm{H}^{+}\right] \\
& \mathrm{dt} \\
& \mathrm{K}_{\mathrm{obs}}=\mathrm{k}_{3} \mathrm{~K}_{2} \mathrm{~K}_{1}[\mathrm{~S}]\left[\mathrm{H}^{+}\right]
\end{aligned}
$$

\section{Oxidation of Unsaturated alcohols}

The kinetics of oxidation of allyl alcohol, crotonyl alcohol and styryl carbinol were investigated at $298 \mathrm{~K}, 303 \mathrm{~K}, 313 \mathrm{~K}, 323 \mathrm{~K}$ and rate constants were calculated as shown in the Table 3 . Using Eyring relation of least square method ${ }^{23}$ the enthalpy of activation $\left(\Delta H^{\#}\right)$, entropy of activation $\left(\Delta S^{\#}\right)$, free energy change $\left(\Delta \mathrm{G}^{\#}\right)$ and Energy of activation $\left(E_{a}\right)$ were evaluated from these temperature range. The entropy of activation was found to be negative for alcohols due to the increase in polarity of the transition state. From the similar values of free energies of activation we can conclude that the oxidation mechanism is same for all the alcohols used as substrate. This is also confirmed from the energy of activation value $E_{a}$, where the value is higher for the slow reaction, oxidation of styryl carbinol $(E a=9.15 \pm 1.2)$. The enthalpies of activation $\left(\Delta \mathrm{H}^{\#}\right)$ values are very low indicating a concerted mechanism as proposed.The iso-kinetic plot ${ }^{24}$ between $\Delta H^{\#}$ versus $\Delta S^{\#}$ gives a straight line (Fig. 5.) with correlation coefficient, $r=0.972$. The iso-kinetic temperature $\beta$ obtained from the slope is $158.38 \mathrm{~K}$. Since the $\beta$ value is lower than the experimental temperature, it indicates that this oxidation is an entropy controlled reaction ${ }^{25,26}$. The Exner plot shows, $r=0.999$, at $303 \mathrm{~K}$ and $313 \mathrm{~K}$ on applying the relation ${ }^{27}\left(\log \left(k_{1}\right) T_{2}=a+b \log \right.$ $\left.\left(k_{1}\right) T_{1}\right)$ where a-intercept, b-slope, (Temperature) $T_{2}>T_{1}$ respectively. This implies that all the alcohols follow the same scheme of mechanism (Figure 6.)

Table 3: Thermodynamic and activation parameters for oxidation of unsaturated alcohols by PzCC

\begin{tabular}{ccccccccccc}
\hline Substrate & Order & \multicolumn{3}{c}{$\begin{array}{c}\mathrm{k}_{1} 10^{4}\left(\mathrm{~s}^{-1}\right) \\
303 \mathrm{~K}\end{array}$} & $313 \mathrm{~K}$ & $\begin{array}{c}\Delta \mathrm{H}^{\#} \\
323 \mathrm{~K}\end{array}$ & $\begin{array}{c}\left.\mathrm{kJ} \mathrm{mol}^{-1}\right) \\
\left(\mathrm{Jk}^{-1} \mathrm{~mol}^{-1}\right)\end{array}$ & $\begin{array}{c}\Delta \mathrm{G}^{\#} \\
\left(\mathrm{~kJ} \mathrm{~mol}^{-1}\right)\end{array}$ & $\begin{array}{c}\mathrm{E}_{\mathrm{a}} \\
\left(\mathrm{kJ} \mathrm{mol}^{-1}\right)\end{array}$ \\
\hline $\begin{array}{c}\text { Allyl } \\
\text { Crotonyl }\end{array}$ & 0.876 & 9.88 & 10.91 & 12.14 & 13.77 & $2.67 \pm 0.6$ & $218.78 \pm 2.8$ & $68.96 \pm 0.8$ & $5.19 \pm 0.7$ & 0.993 \\
Styryl carbinol & 0.882 & 25.84 & 30.1 & 37.11 & 46.6 & $5.63 \pm 0.8$ & $205.27 \pm 3.5$ & $67.83 \pm 1.0$ & $8.15 \pm 1.1$ & 0.991 \\
\hline
\end{tabular}

$[$ Alcohols $]=1.5 \times 10^{-2} \mathrm{~mol} \mathrm{dm}^{-3} ;[\mathrm{PzCC}]=2.0 \times 10^{-3} \mathrm{~mol} \mathrm{dm}^{-3} ;\left[\mathrm{H}^{+}\right]=1.0 \times 10^{-1} \mathrm{~mol} \mathrm{dm}^{-3}$

\section{Structural activity of alcohols}

The resonance stabilisation and the positive character of styryl carbinol is higher than crotonyl and allyl alcohol and hence the reactivity is believed to be in the order of Allyl alcohol< Crotonyl alcohol< Styryl carbinol. The electron cloud is higher in the styryl carbinol and hence the activation energy is also higher $\left(E_{a}=9.15 \mathrm{KJ} \mathrm{mol}^{-1}\right)$. 


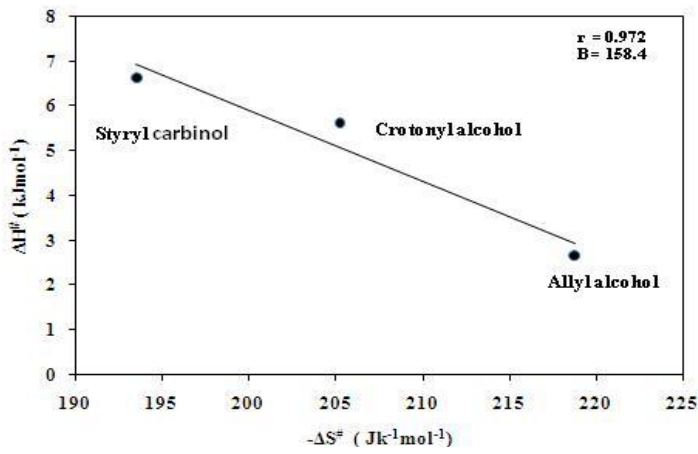

Fig. 5. Isokinetic plot of $\Delta \mathbf{H}^{*}$ versus $-\Delta \mathbf{S}^{\#}$

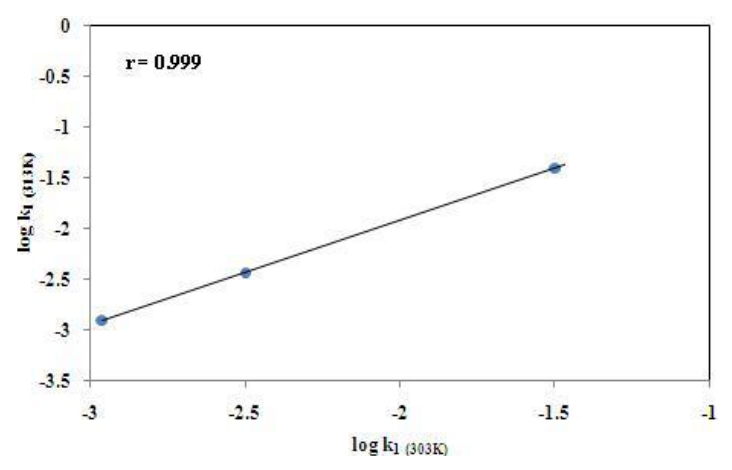

Fig. 6. Exner plot of $\log k_{1(313 \kappa)}$ versus $\log k_{1(303 K)}$

\section{CONCLUSION}

The reaction was first order with respect to [Alcohols], [PzCC] and [Perchloric acid]. The stoichiometry was found to be 1:2 that is one mole of substrate consumed two moles of oxidant. The activated complex formed was rigid due to the negative $\Delta S^{\#}$ value. The polymerisation was not observed due to absence of free radicals. The rate constant values of the oxidant $[\mathrm{PzCC}]$ was decreased due to the presence of $\mathrm{Cr}^{+6}$ as acidic chromate and due to the electron withdrawing nature of two nitrogen groups in the pyrazine ring. The order of reactivity was [Allyl alcohol] $<$ [Crotonyl alcohol] $<$ [Styryl carbinol]. From the kinetic data the plausible mechanism has been proposed and the suitable rate law is derived.

\section{ACKNOWLEDGEMENT}

This research work was carried in the Center for Research, Arignar Anna Government Arts College, Musiri, Trichy. I thank all the Faculties, Research Scholar for their support.

\section{REFERENCES}

1. Sheldon, R. A.; Kochi, J. K.; Metal catalyzed oxidation of organic compounds, Academic Press, New York., 1981.

2. Fieser, L.; Fieser, M.; Reagents for organic synthesis, New York, John Wiley and Sons., 1967.

3. House, H. O.; Benjamin, W. A.; Modern synthetic reactions, Inc. Menlo Park, CA., 1972.

4. Lucia Tonucci,; Marco Nicastro,; Nicola d'Alessandro,; Mario Bressan,; Primiano D’Ambrosio,; Antonino Morvillo.; Green Chem., 2009, 11, 816-820.

5. Cainelli, G.; Caridillo, G.; Chromium oxidation in organic chemistry, Springer-Verlag, Berlin., 1984.

6. Saleem, M. D.; Nirmal, N. H.; Sharanappa, T.N.; Transition Met. Chem., 2002, 27, 207-212.

7. Hiran, B.L.; Jain, S.; Bhatt, C.V.; E-J Chem., 2009, 6, 237-246.

8. Palaniappan, AN.; Sekar, K .G. ; Ravishankar, M.; Oxid. Commun., 1995, 18, 52 - 55.

9. Kabilan, S.; Vetrichelvan, K.; Sankar, P.;
Krishnasamy, K.; Afinidad. L1., 1994, 169-172.

10. Aizpurua, J.M.; Juaristi, M.; Lecea, B.; Palomo, C.; Tetrahedron., 1985, 41, 2903-2911.

11. Chimatadar, S.A.;Koujalagi, S.B.;Nandibewoor, S. T.; Oxid. Commun., 2004, 27, 81 - 89.

12. Sekar, K. G.andVellaisamy, M. Kinetics of oxidation of allyl alcohol by imidazolium dichromate. Der Chemica Sinica., 2012, 3, 703-707.

13. Seplapatty Kalimuthu Periyasamy;Krishnamoorthy Gunasekar; ILCPA., 2012, 5, 8-19.

14. Degirmenbas, N.; Ozgun, B.; Monatshefte für Chemie., 2004, 135, 407-410.

15. Choudhary, K.; Sharma, P. K.; Banerji. K. K. Int. J. Chem. Kinet., 1999, 31, 469-475.

16. Rajalakshmi, K.; Ramachandramoorthy, T.; Srinivasan, S.; J. Chem. Pharm. Res., 2012, 4, 894-900.

17. Davis, H. B.; Sheets, R. M.; Brannfors, J. M.; Paulder, W. W.; Gard, G. L.; Heterocycles, 1983, 20, 2029. 
18. Feigl, F.; Spot Tests in Organic Analysis, Elsevier, Amsterdam., 1966, 482.

19. Krishnapillay, A.; Thirunavukkarasu, A.; Indian J. Chem., 1981, 20, 583-585.

20. Wiberg, K.B.; Oxidation in Organic Chemistry, PartA, Academic Press, Newyork., 1965.

21. Sekar, K. G.; Prabakaran, A.; Oxid. Commun., 2008, 31, 348-355.

22. Banfi, S.; Cavazzini, M.; Pozzi, G.; Barkanova, S. V.; Kaliya, O. L.; J. Chem.Soc. Perkin Trans.,
2000, 2, 871-877.

23. Eyring, H.; J. Chem. Phys., 1935, 33, 107-115.

24. Leftler, J. E.; J. Chem. Phys., 1955, 23, 2199-2200.

25. Saradamani, P.R.; Jegannathan, V.; Indian J. Chem., 29A, 1990, 1, 700-702.

26. Kirubasankar, J. Ph.D. Thesis, Annammalai University., 1998.

27. Palm, V.A.; Vizgert, R.V.; Pokl Akad Nauk USSR., 1962, 142, 1091-1096. 